\title{
Comparative Study of Pitch-Plunge Airfoil Aerodynamics at Transitional Reynolds Number
}

\author{
Yongsheng Lian*, Michael V. OL ${ }^{\dagger}$, Wei Shyy
}

This paper describes a combined numerical analysis and experimental study of flow characteristics on a pitching-plunging SD7003 airfoil at the transitional Reynolds number of 60,000. For experiments, we use particle image velocimetry to reveal the detailed flow structure at the vicinity of regions of interest. For numerical simulation, we solve the incompressible Navier-Stokes equations on a moving grid using a pressurebased method. Three models are used to simulate the transitional flows: the shear-stress transport model, an $e^{N}$ type transitional model, and a detached eddy simulation model. The solutions are compared with both phaseaveraged and snapshot experimental results. The motion under consideration is motivated by kinematics for maximum propulsive efficiency, with plunge-induced angle of attack partially cancelling the pitch, resulting in small excursions in angle of attack past static stall. Nevertheless there are significant post-stall effects, with individual velocity field snapshots differing markedly from the phase average.

\section{Nomenclature}

$C_{D} \quad=$ Drag coefficient per unit span

$C_{L} \quad=$ Lift coefficient per unit span

$c \quad=$ Airfoil chord $(=152.4 \mathrm{~mm})$

$f \quad=$ physical frequency of airfoil oscillation

$h_{0} \quad=$ non-dimensional plunge amplitude

$k \quad=$ reduced frequency, $\omega \mathrm{c} / 2 U_{0}$

St $\quad=$ Strouhal number, $2 f c h_{0} / U=2 k h_{0} / \pi$

$U_{0} \quad=$ reference velocity, typically freestream velocity

$\alpha \quad=$ kinematic angle of attack

$\alpha_{0} \quad=$ mean angle of attack

$\alpha_{e} \quad=$ effective angle of attack

$\alpha_{g} \quad=$ geometric angle of attack

$\beta \quad=$ kinematic angle of attack due to pitching motion

$\eta \quad=$ propulsive efficiency

$\theta \quad=$ pitching angle of attack

$\varphi \quad=$ Phase of motion, in fractions of one period

$\psi \quad=$ phase difference between pitching and plunging

$\omega \quad=$ pitch/plunge circular frequency

\section{Introduction}

One can elucidate two kinds of perspectives in recent work on low Reynolds number unsteady aerodynamics for Micro Air Vehicle (MAV) applications. The first, espoused principally by zoologists and designers of practical flapping-wing MAV flight articles, involves mechanical models that attempt to closely represent specific examples in nature [1-5]. For natural flyers, the motion kinematics tends to be complex, and difficulty in either measurement or computation tends to lead towards a descriptive approach. The second, to which the present work belongs, studies aggressive abstractions such as the pitching/plunging 2D airfoil, in an effort at isolating canonical problems for parametric study of effects of Reynolds number, reduced frequency, reduced amplitude and so forth. The simplification favors detailed investigation, but leaves open the issue of

\footnotetext{
* Research Specialist, Department of Aerospace Engineering, University of Michigan, 1320 Beal Ave, Ann Arbor, MI 48109, AIAA Member, ylian@umich.edu

${ }^{\dagger}$ Aerospace Engineer, Air Vehicles Directorate, AFRL/VAAA, Bldg. 45, 2130 8th St., Wright-Patterson AFB, OH 45433-7542; AIAA Associate Fellow, Michael.Ol@wpafb.af.mil

"Clarence L. "Kelly" Johnson Collegiate Professor and Chair, Department of Aerospace Engineering, University of Michigan, 1320 Beal Ave, Ann Arbor, MI 48109, AIAA Fellow, weishyy@umich.edu
} 
direct aid to aerodynamic design of MAVs. The prescription of "two dimensions" is itself rife with ambiguities, such as the role of wind tunnel/water tunnel test section walls in producing $3 \mathrm{D}$ effects, of which a typical example is spanwise flow in shed vortices in the wake [6]. With increasing reduced frequency of motion, 3D effects can dominate vortex shedding in the immediate vicinity of the airfoil [6], presumably with important consequences for the time-dependent aerodynamic loads. Nevertheless, with appropriate treatment, largely 2D flow field can be established in certain conditions, which allows one to simplify experimental-computational comparison in specific problems such as transitional flows and massive shedding of vortical structures.

The combination of low Reynolds number $\left(\operatorname{Re}<10^{5}\right)$ phenomena and high substantial flow structure variations encountered in MAV-relevant flows suggest departure from classical unsteady airfoil theory [7]. Crucial issues include the role of leading edge and trailing edge vortex shedding, interaction of the timedependent wing pressure distribution with vortices shed previously during the motion time history, and the role of transition in shear layers bounding regions of laminar separation. Prior to MAVs, dynamic stall of helicopter blades was perhaps the main application for high-rate unsteady aerodynamics in nominally two dimensions, but the difference in Reynolds number is a factor of on the order of 100. It was established that the dominant feature of dynamic stall is the formation and shedding of a strong vortex-like disturbance from near the leading-edge. McCroskey et al [8] pointed out that as the vortex passes over the airfoil surface it significantly changes the chordwise pressure distribution and produces transient forces and moments that are fundamentally different from those in static stall. Comprehensive reviews of dynamic stall and unsteady airfoils are given by McCroskey [9], Carr [10], and Carr and McCroskey [11]. Ohmi et al. [12,13] experimentally examined the starting flows past a 2D oscillating and translating airfoil. They found that the reduced frequency is the dominant parameter of the flow. However, they also demonstrated that as the pitching frequency increases, the patterns of the vortex wake are depended on both the reduced frequency and the amplitude. Visbal and Shang [14] performed numerical investigation of flow structure around a rapidly pitching NACA0015 airfoil at Reynolds number of $10^{4}$ by solving the full 2D Navier-Stokes equations. They observed a strong dependence of the primary flow features on the pitch rate and pitch axis position. At a fixed axis position, the dynamic stall can be delayed with increased pitch rate. Ghosh Choudhuri and Knight [15] examined the effects of compressibility, pitch rate, and Reynolds number on the initial stages of 2D unsteady separation of laminar subsonic flow over a pitching airfoil in the Reynolds number ranging from $10^{4}$ to $10^{5}$. They discovered that increasing the Reynolds number hastens the appearance of the primary recirculating region.

The aforementioned studies focus mostly on transients upon initiation of airfoil motion from rest. Others considered the periodic or phase-averaged behavior of pitch/plunge after transients have relaxed, typically with a focus on motion kinematics for optimal thrust efficiency. Platzer and Jones [16] discussed theoretical prediction of thrust efficiency vs. flow visualization and thrust measurements for an airfoil in pure-plunge, over a range of reduced frequencies, $k=\omega c / 2 U_{\infty}$, and reduced amplitude, $h=1 / c$, where 1 is the one-sided plunge amplitude. Young and Lai [17] used a 2D Reynolds-Averaged Navier-Stokes (RANS) approach to study the $(k, h)$ parameter space for optimal thrust efficiency. Lian and Shyy [18] used RANS methods to study the effect of an abstraction of gusts on a pitching/plunging airfoil, with evidence that the flapping motion has gust load alleviation potential, and that gusts can cause hysteresis in the force history and affect the transition process.

In this paper we compare experiment and computation for a $2 \mathrm{D}$ airfoil experiencing sinusoidal pitch/plunge motion at the "transitional" Reynolds number of 60,000 , focusing on a cambered airfoil that is nominally on-design - the SD7003 [19]. We compare a case of motion kinematics where the total effective angle of attack passes slightly beyond the point of static stall, with a case essentially in deep stall.

The kinematics of airfoil harmonic pitch-plunge (Figure 1) can be written as follows:

$$
h(t)=h_{0} c \cos \left(\omega t+\phi_{h}\right) \quad \theta(t)=\theta_{0} \cos \left(\omega t+\phi_{\theta}\right)+\alpha_{0}
$$

where $h_{0}$ is the plunging amplitude non-dimensionalized by the airfoil chord $c, \theta(t)$ is the pitching motion with an amplitude of $\theta_{0}$, a frequency of $\omega$, and a mean angle of attack of $\alpha_{0}$. The flapping motion $h_{0}$ is defined positive upward and pitch amplitude positive clockwise. The leading angle between pitch and plunge is $\psi=\phi_{\theta}-\phi_{h}$. As usual, the plunge and pitch frequency are equal. $k=\omega \mathrm{c} / 2 U_{0}$ is the reduced frequency, and $S t=2 \mathrm{kh} / \pi$ is the Strouhal number, defined following Anderson et al. [20]. 


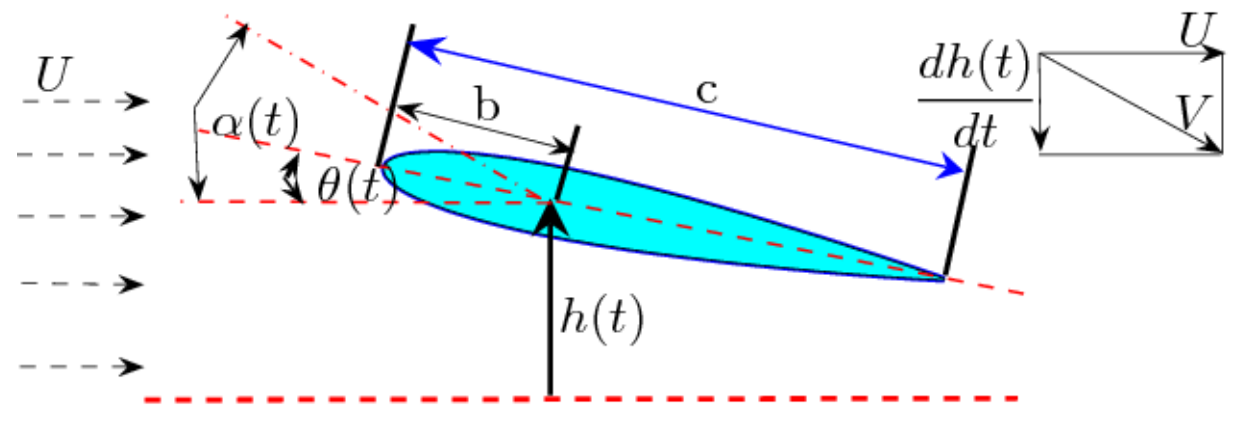

Figure 1. Nomenclature for airfoil pitch-plunge.

There are two length scales, chord $c$, and stroke amplitude $c h_{0}$. Thus both $S t$ and $k$ are required to describe the relative time and length scales. The kinematic angle of attack is relative to the pivot point and is defined as:

$\alpha(t)=\theta(t)+\beta(t)=\theta(t)+\tan ^{-1}\left(-\frac{1}{U_{0}} \frac{d h(t)}{d t}\right)=\theta_{0} \cos \left(\omega t+\phi_{\theta}\right)+\tan ^{-1}\left(\pi \operatorname{St} \sin \left(\omega t+\phi_{h}\right)\right)$

\section{Mathematical Models and Numerical Methods}

The computational solver is an in-house code that solves the Navier-Stokes equations on a moving grid with a pressure-based algorithm [21]. We use Menter's shear stress transport (SST) model [22] as the turbulence closure model:

$$
\begin{aligned}
& \frac{D \rho k}{D t}=\tau_{i j} \frac{\partial u_{i}}{\partial x_{j}}-\beta^{*} \rho \omega k+\frac{\partial}{\partial x_{j}}\left[\left(\mu+\sigma_{k} \mu_{t}\right) \frac{\partial k}{\partial x_{j}}\right] \\
& \frac{D \rho \omega}{D t}=\frac{\gamma}{v_{t}} \tau_{i j} \frac{\partial u_{i}}{\partial x_{j}}-\beta \rho \omega^{2}+\frac{\partial}{\partial x_{j}}\left[\left(\mu+\sigma_{\omega} \mu_{t}\right) \frac{\partial \omega}{\partial x_{j}}\right]+2 \rho\left(1-F_{1}\right) \sigma_{\omega 2} \frac{1}{\omega} \frac{\partial k}{\partial x_{j}} \frac{\partial \omega}{\partial x_{j}}
\end{aligned}
$$

The eddy viscosity can be obtained by applying a blending function, $F_{2}$ :

$$
v_{t}=\frac{a_{1} k}{\max \left(a_{1} \omega, \Omega F_{2}\right)}
$$

This model maintains robust and accurate formulation of the Wilcox $k-\omega$ model in the near-wall region and fully retains the freestream independence of the k- $\varepsilon$ model. For the detailed explanation of each term and the definition of each parameter we refer to Menter [22]

For the Navier-Stokes equations, the convection terms are discretized with the second-order upwind scheme and the diffusion terms are discretized with the second-order central difference scheme. The time integration is performed with a second-order implicit three-point backward scheme for better handling of accuracy and strict time step constraint imposed by the extremely fine grid resolution. Three models are used for the transitional flow simulation characterized with strong vertical flows. They are the Menter's SST model [22], SST model with a modified empirical transition method [23] based on the principle of the $\mathrm{e}^{N}$ method [24,25] and the work of Drela and Giles [26], and the detached eddy simulation (DES) based on the SST turbulence model [27]. The SST model is chosen because its detective capability and responsiveness to flow separation in adverse pressure 
gradient [28]. Instead of solving the Orr-Sommerfel equation to evaluate the local growth rate of the unstable waves based on the velocity, the empirical transition model approximate the growth rate by line integration. A DES is a three-dimensional unsteady numerical simulation based on a turbulence model. In this approach, the flow filed is solved directly in regions where grid density is fine enough and the flow field is modeled in regions where it is not. It is essentially a hybrid approach of LES and RANS computations. To eliminate the gridmovement-related errors due to the wing motion, the geometric conservation law [29] is implemented .

The boundary consists of three types: inlet, outlet, and airfoil surface (Figure 2). At the inlet, we assign the Dirichlet boundary condition, i.e., the velocity is equal to the freestream velocity; at the outlet, we set the pressure to zero and assign a zero-gradient condition for the velocity; on the airfoil surface we use the nonslip boundary condition for the velocity and approximate the normal pressure gradient from the momentum equation,

$$
\partial p / \partial \mathbf{n}=-\rho \mathbf{a} . \mathbf{n}
$$

Where $\mathbf{n}$ is the vector of the normal direction of the airfoil surface and $\mathbf{a}$ is the acceleration on the airfoil surface.

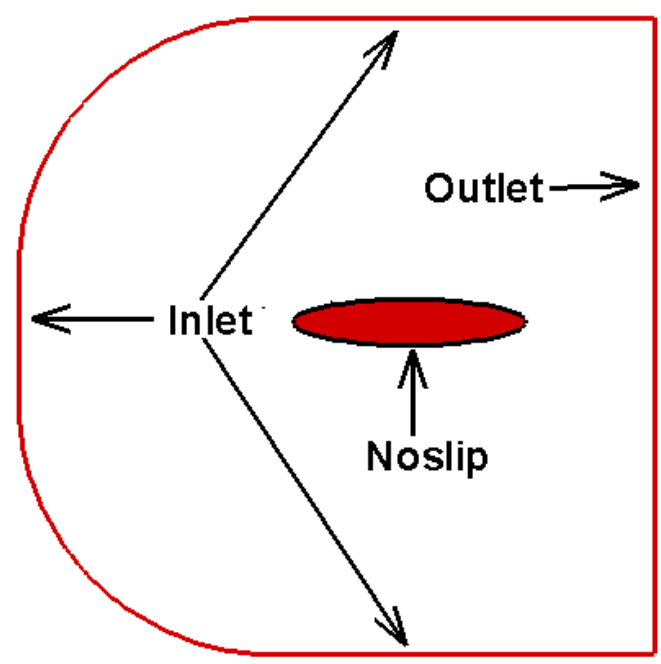

Figure 2. Schematic of the boundary conditions. Outer boundaries are 30-chord away from the airfoil.

\section{Experimental Setup}

Experiments were conducted in the Air Force Research Laboratory's Horizontal Free-surface Water Tunnel ("HFWT"); the HFWT and the approach used for airfoil PIV measurements are described in Ol et al.[30]. PCO 400011 Mpix cameras [31] were triggered off of an external pulse train derived from the position encoder of the motion rig, thus allowing for selection of motion phase at which to acquire data. Airfoil motion was driven by a 2-degree-of-freedom "pitch-plunge rig", consisting of a pair of electric linear motors mounted vertically on a plate above the tunnel test section free-surface. Each motor actuates a vertical rod, which connects via a bushing to the airfoil at a fixed pivot point on the airfoil chord. Motion trajectory of each rod is programmed independently, allowing for single degree-of-freedom motions such as sinusoidal pure-pitch or pure-plunge, as well as nontrigonometic and combined motions. The pivot point for pitching motions can be varied as well, but for simplicity was limited in the present study to the quarter chord. Details of the pitch-plunge rig are given in Ol [32].

Velocity data were acquired at four phases in each period of oscillation, for two sequences of 120 periods each. Prior results with dye injection and PIV for pure-pitch at $k=3.93$ and $\operatorname{Re}=60,000$ show that startup transients relax in less than two periods of oscillation after startup, where the startup is continuous in velocity and bounded 
by $5 \mathrm{~m} / \mathrm{s}^{2}$ in acceleration in the controller software. In the present work, the first 5 periods were removed from each image sequence, and the remaining 230 velocity records for each phase of motion were averaged. Vorticity was calculated by explicit differentiation of cubic spline fits to the velocity field [30].

Resolution was 88 pixels/cm. For $32 \times 32$ pixel windows with $16 \times 16$ overlap, this results in 84 velocity vectors per chord length. Because of laser reflections from the model surface (polished stainless steel, painted flat-black) and lack of corrections for PIV windows which intersect with the model surface or for near-wall pixel regions saturated due to laser reflections, data closer than one window length to the airfoil wall - approximately $0.02 \mathrm{c}-$ are not reliable. This renders problematic a direct comparison of PIV and CFD in attached boundary layers, but offers sufficient detail to make comparisons regarding shear layers bounding laminar separation bubbles, and shed vortical structures.

The installation of the model and schematic of the rig assembly are shown in Figure 3. In the photograph, the model is inside the test section, but the glass walls are not visible. In the schematic, the pitch/plunge rig's linear motors are atop a plate above the test section's free surface, and the test section is not shown.
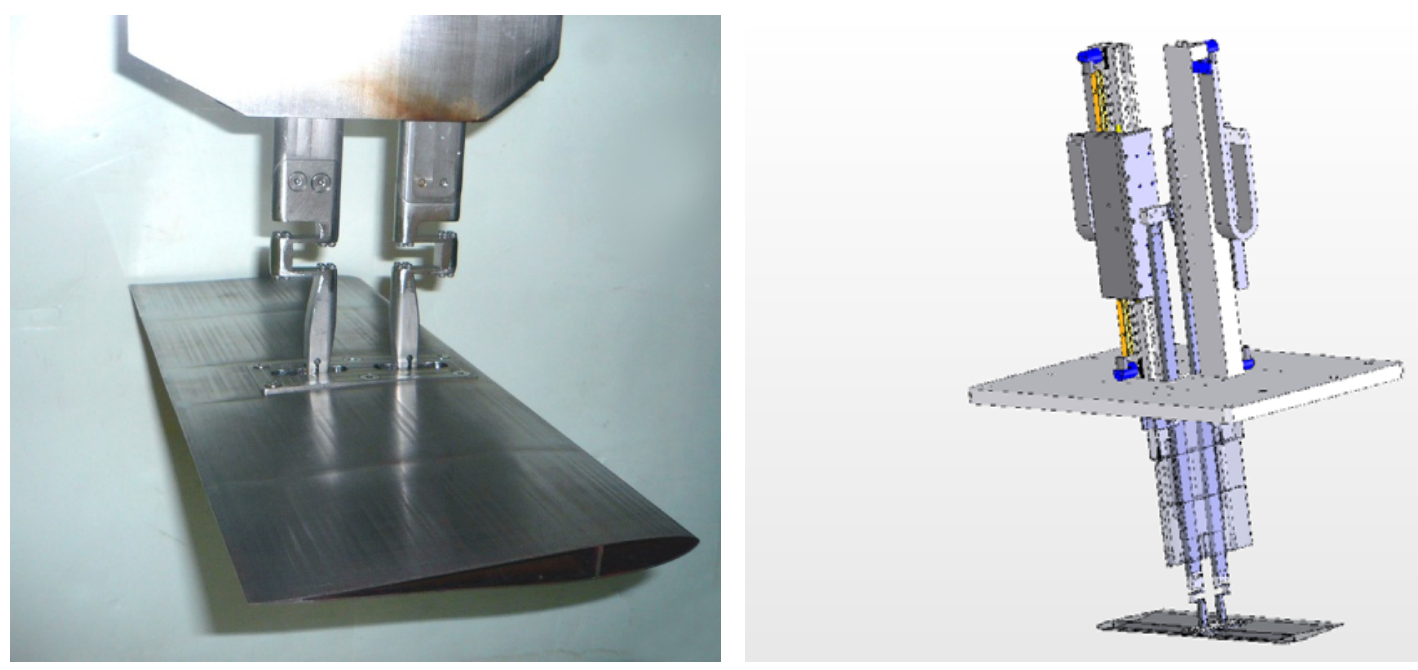

Figure 3. SD7003 airfoil installed in HFWT test section, with plunge rods in position prior to a run (left); and schematic of pitch/plunge rig and airfoil model (right).

\section{$\underline{\text { Results and Discussion }}$}

\section{Problem description}

The first case under consideration has pitch leading plunge by $90^{\circ}, \alpha_{0}=8^{\circ}, \theta_{0}=8.42^{\circ}, h_{0}=0.5$, and $k=0.25$. The motivation follows pitch-plunge scenarios of high propulsive efficiency in $2 \mathrm{D}$ flapping [20]. Since the SD7003 airfoil has static stall at $\mathrm{Re}=60,000$ at around $11^{\circ}$ [30], and the peak effective angle of attack is $13.6^{\circ}$ (attained at phase $\varphi=0.25$ ), this should be an example of weak dynamic stall. The second case was a pureplunge version of the first. Here, the pitch-geometric alpha is not available to partially cancel the plunge-induced alpha, so the extremes in total angle of attack are larger, with deeper excursion past static stall. The two cases, with motion time history as recorded from the plunge rig's optical encoder tape, are given in Figure 4. Both cases are cosine-waves in plunge. Minor oscillations in the encoder signal are expected to be absorbed by elasticity of the plunge rods and the airfoil mount.

For $\operatorname{Re}=60,000, k=0.25$ corresponds in the experiment to a physical frequency of $0.206 \mathrm{~Hz}$ and tunnel flow speed of $39.4 \mathrm{~cm} / \mathrm{s}$. With a $61 \mathrm{~cm}$ wide by $61 \mathrm{~cm}$ high test section, blockage based on projected frontal area of the model at maximum pitch amplitude is $6 \%$. Gaps between the model tips and the tunnel walls were approximately $1.0 \mathrm{~mm}$. To obtain the "most 2-dimensional" flow field, the PIV light sheet was placed at the 3/4 span location; 
that is, approximately halfway between the plunge rods and the tunnel wall. Light sheet thickness was approximately $2 \mathrm{~mm}$, though the large field of coverage (up to $45 \mathrm{~cm}$ ) makes precise collimation of the light sheet difficult.
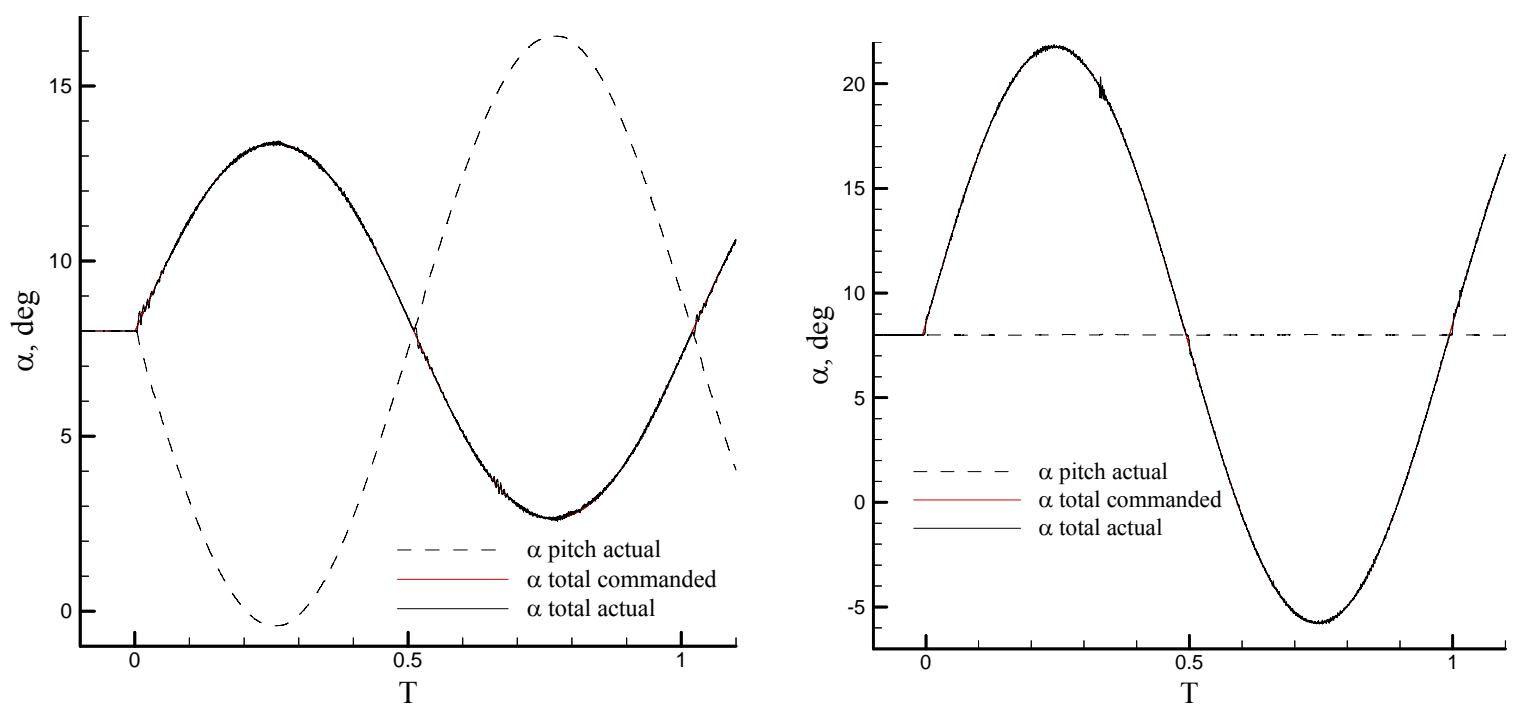

Figure 4. Time-traces of effective total angle of attack (solid line) and pitch angle (dashed line), from linear motor encoders: pitch-plunge case (left) and pure-plunge case (right).

\section{Computational sensitivity analysis}

To assess the impact of spatial and temporal resolution in the computation, the number of grid points in the streamwise and radial directions was varied, resulting in six different grid systems ranging from $220 \times 90$ to $600 \times 250$. For all the grids, we used the same hyperbolic tangent distribution in the radial direction: the first grid is $6.7 \times 10^{-5}$-chord away from the airfoil, and the last grid is 1.3-chord away from the outer boundary. For the $410 \times 200$ grid, we estimate that there are about 40 to 50 grid points inside the boundary layer. Figure 5 compares amongst the three grids the lift and drag histories of the first period after motion startup, for the SST model. The maximum difference in $\mathrm{c}_{1}$ between the coarse grid and medium grid is around $4 \%$ near $\mathrm{t} / \mathrm{T}=0.6$. In other regions, the discrepancy is less than $0.5 \%$. The discrepancy between the fine and medium grid is well below $1 \%$ in all regions. This observation is also true for the drag history. The flow structures, as illustrated from the streamwise velocity contour plots in Figure 6, are in good mutual agreement amongst the three grids. The medium grid of 410x200 seems sufficient for the RANS model computation. Our computations based on the transition model also show that the $410 \times 200$ grid is sufficient. Therefore, the medium grid is then used throughout this research. Examining temporal resolution effects, our tests show that sufficient temporal resolution can be obtained with 400 time steps per flapping cycle. We do not pursue a grid-dependent solution for the DES-type simulations.

\section{RANS computations of the pitch-plunge case}

With RANS computations without the transition model (Figure 7), the lift and drag coefficient history attains periodic behavior after the fourth cycle, whereupon the lift in particular closely follows the time trace of dynamic angle of attack. However, when the transition model is included, the force time history shows more variation. Focusing for example on the fifth cycle of motion, lift continues to increase after the maximum dynamic angle of attack is attained, and then quickly drops. The time-averaged lift coefficient of the SST computation without transition model is 0.85 and 0.80 , respectively. 

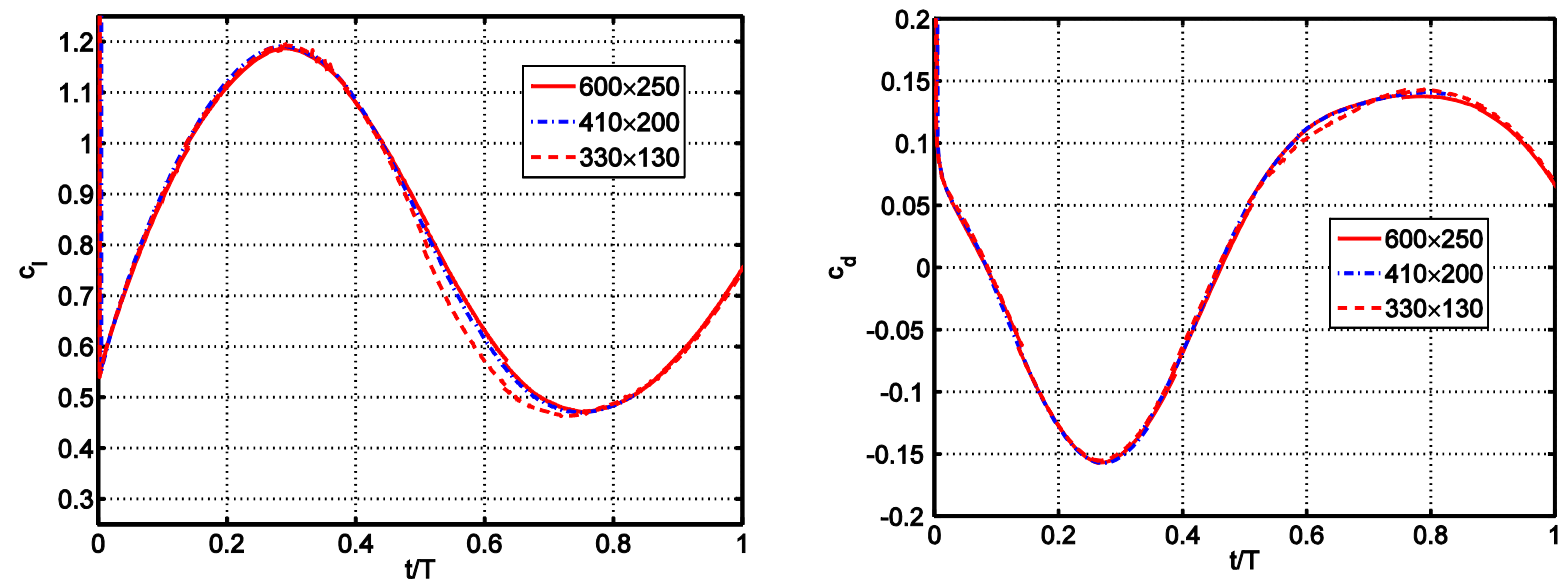

Figure 5. Effect of spatial resolution on the aerodynamic forces for the pitch-plunge case, over one period of motion. Left: lift coefficient; right: drag coefficient.
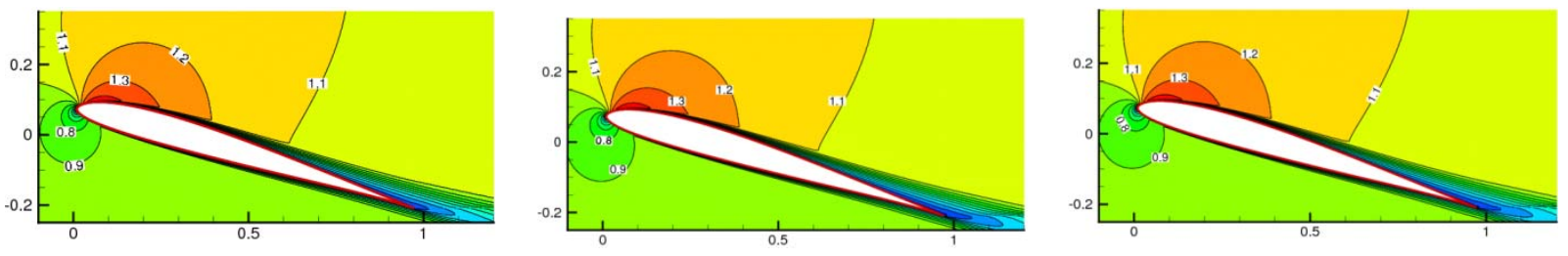

Figure 6. Effect of spatial resolution on the velocity distribution at $\varphi=0.75$. From left to right are grid sizes of: 330x160, 410x200, and 600x250.

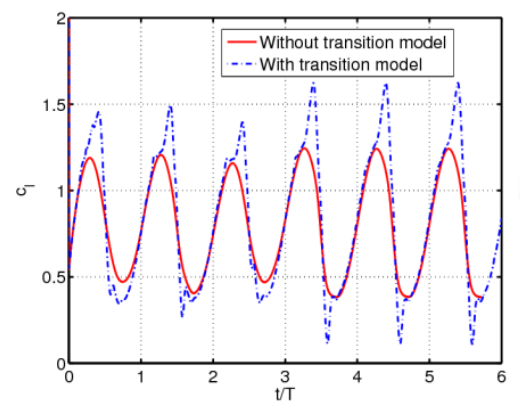

(a)

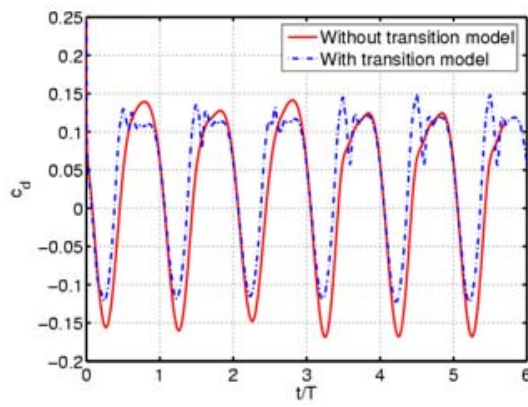

(b)

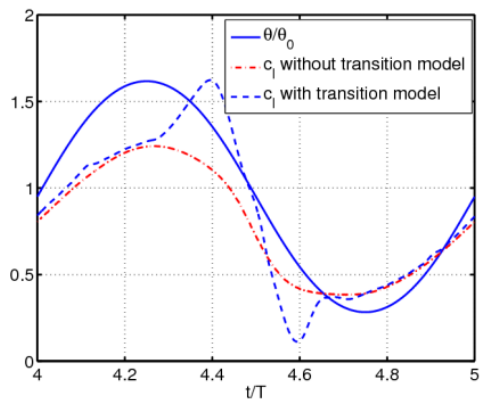

(c)

Figure 7. Comparison of lift and drag coefficient histories for computations with the SST model, with without the transition model.

Figure 8 compares the velocity contours at four phases of motion, among the experiment and RANS computations. At $\varphi=0$, experiment and SST computation show that flow remains attached but SST with the transition model predicts a thin separation bubble covering about $25 \%$ of the airfoil upper surface. Due caution should be paid to interpret the near wall data of the experiment, as mentioned above. At $\varphi=0.25$, both experiment and SST computation show flow separation near the trailing edge. With the transition model, the computation predicts a large and thick separation bubble. The bubble continues to grow in size and creates a low pressure zone on the top surface. Consequently, the lift continues to increases after the airfoil passes its static stall angle of attack until the bubble bursts near $\varphi=0.4$. The difference among the experiment and computations is considerable at $\varphi=0.5$ : the experiment shows in the phase average a separation bubble closing at the trailing edge, while computations predict that flow does not reattach. At $\varphi=0.75$, only mild separation is observed in both the experiment and the computations, with the computations showing slightly more separation than the experiment. 


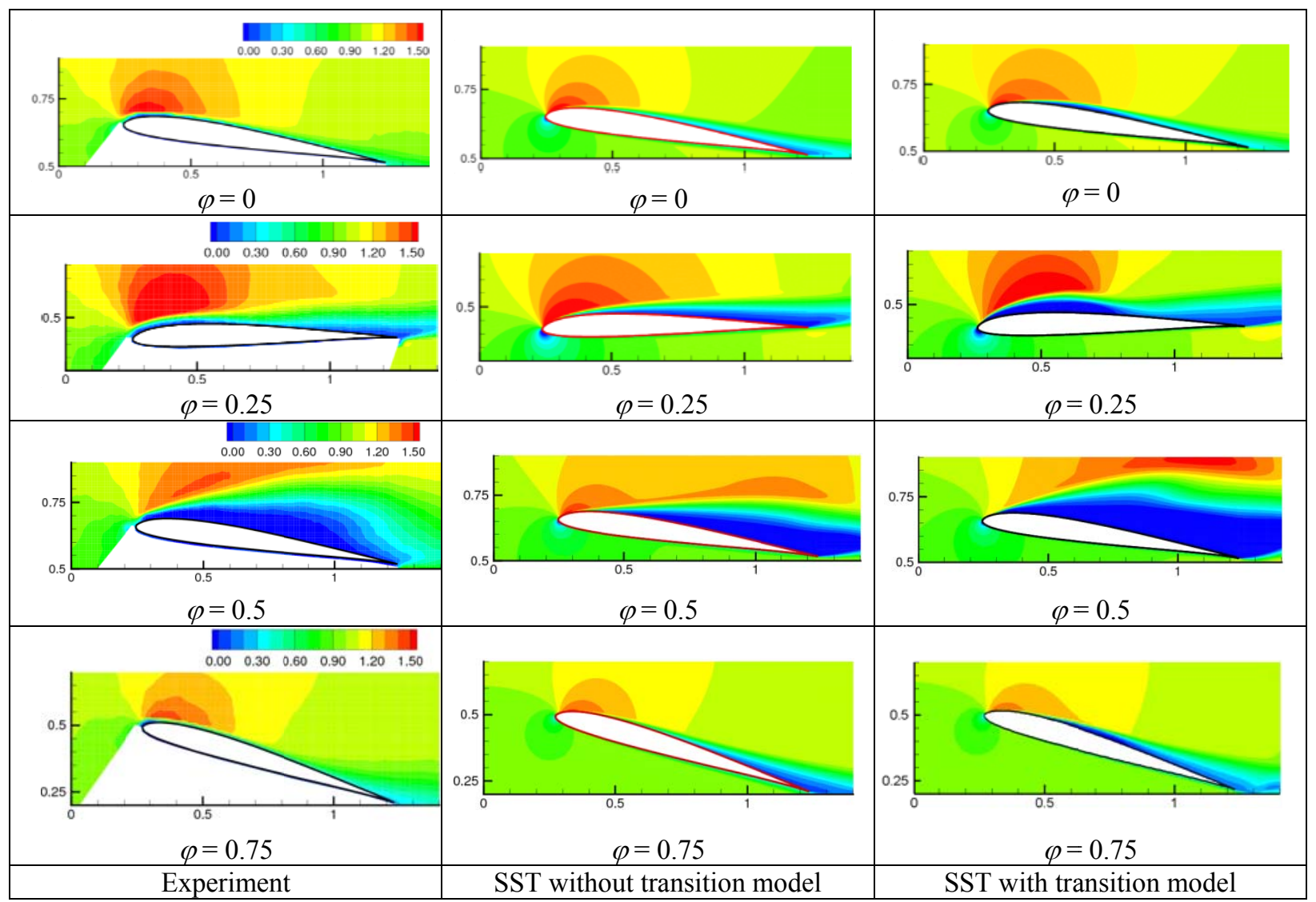

Figure 8. Comparison of normalized streamwise velocity contours at four phases for the pitch-plunge case. Contour levels are from zero to $1.5 U_{0}$.

Normalized streamwise velocity profiles at one-chord away from the trailing edge are shown in Figure 9; velocity measurement further downstream is not possible because of the limits of the PIV field of view. Examination of streamwise-component velocity profiles is useful in estimating whether drag or thrust is generated at the given phase, but extrapolation to time-averaged net drag or thrust from phase snapshots is a tenuous proposition. Also, unsteady effects and pressure-relaxation effects are ignored. In all four phases the profile shows a wake pattern, suggesting net drag. There is no force measurement in the experiment. The time-averaged drag coefficient in the computation with and without transition is 0.040 and 0.016 , respectively. The larger drag with the transition model is mainly due to the separation bubble present from $\varphi=0.25$ to 0.4 . Overall, both computations predict a much lower velocity deficit than the experiment in all four phases, but at $\varphi=0.75$ agreement between experiment and the computation with transition is coincidentally quite close.

The vorticity contours are shown in Figure 10. At $\varphi=0.25$ and 0.5 , the SST computation with the transition model could be argued to evince a leading edge vortex - whereas the experiment and computation without turbulence model evidently do not. In fact, the vorticity contour plots suggest that overall the computation without transition model matches better with experiment. 


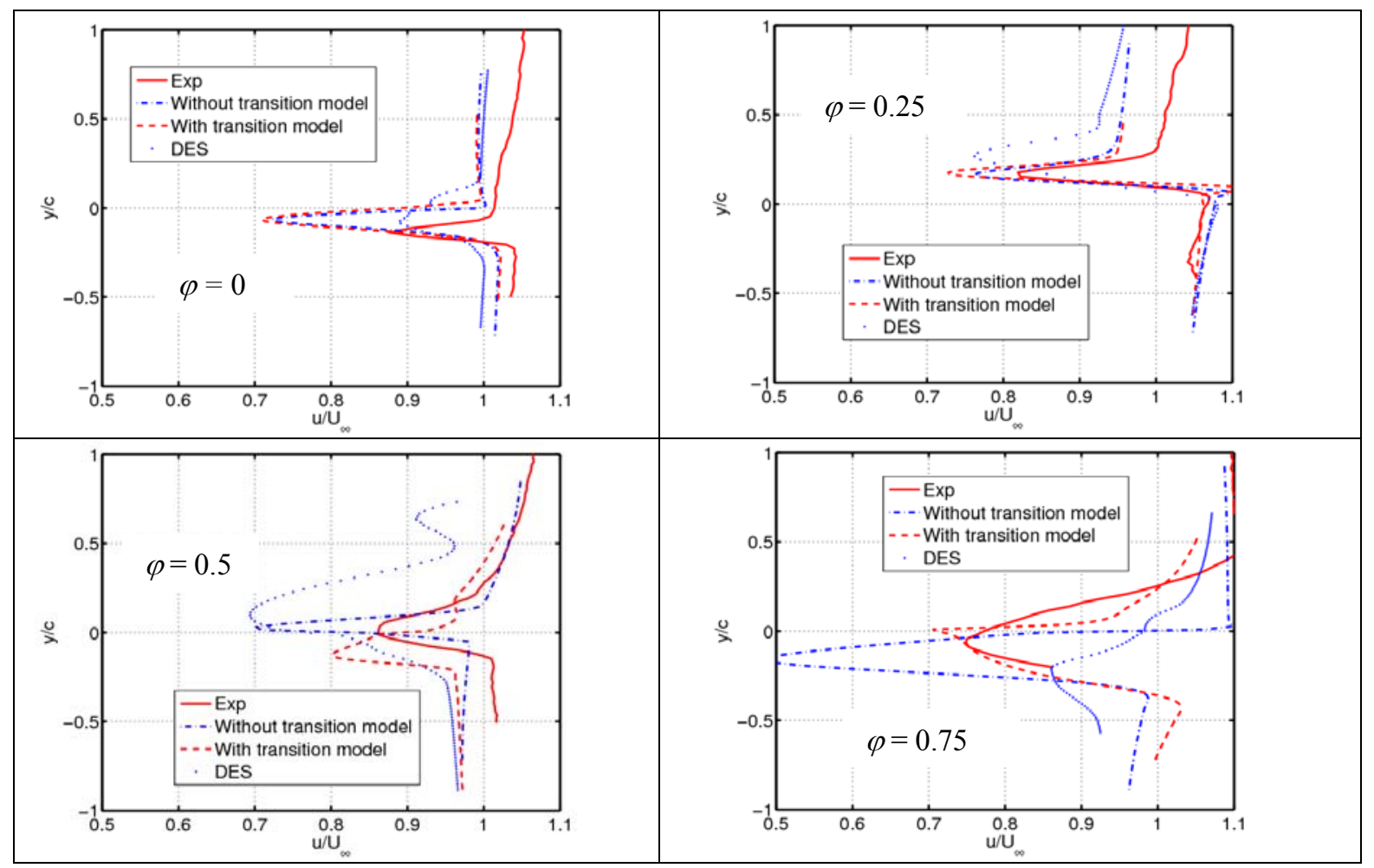

Figure 9. Streamise momentum-wake velocity profiles for the pitch-plunge case.

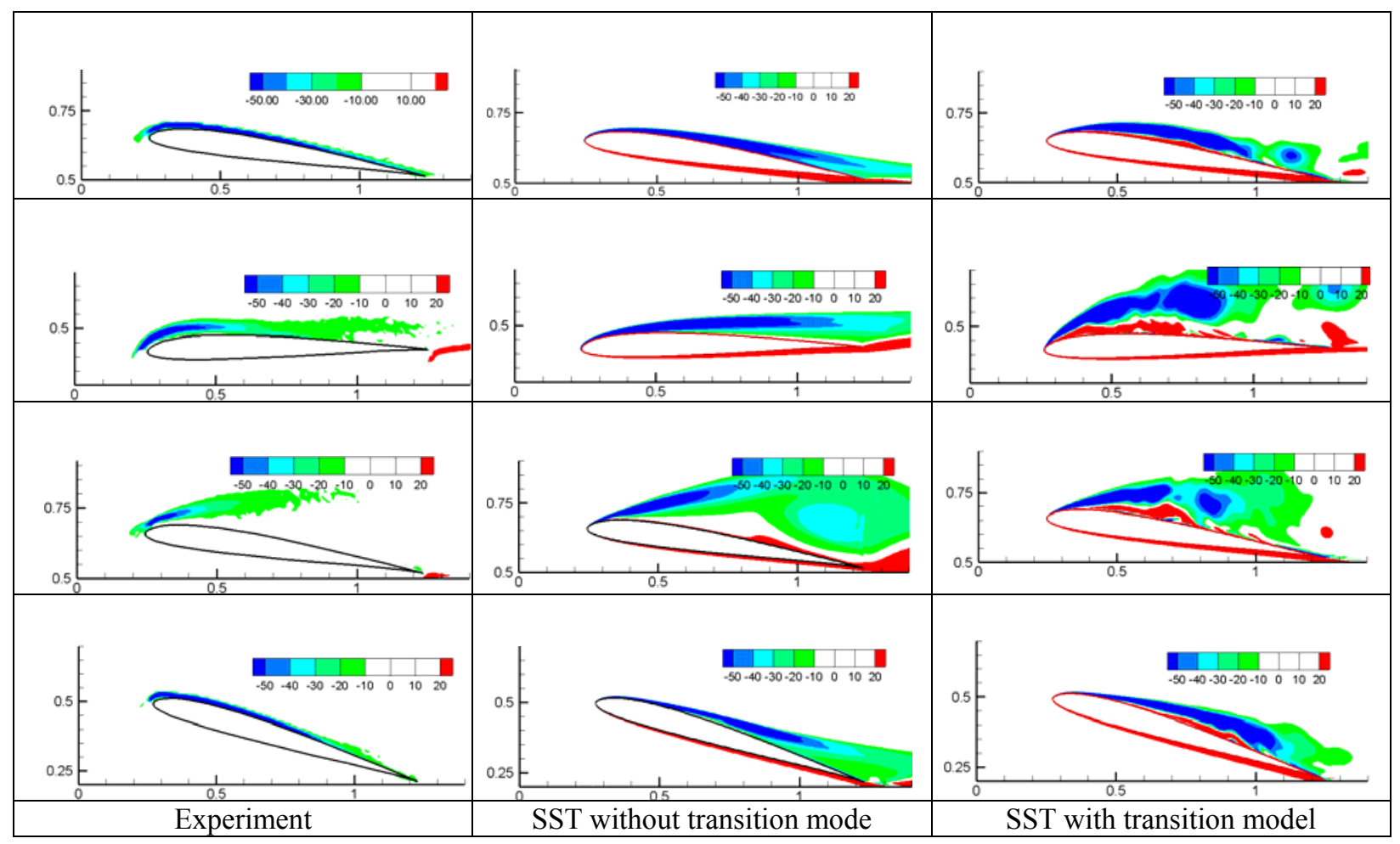

Figure 10. Vorticity contours for the pitch-plunge case. From top to bottom: $\varphi=0,0.25,0.5,0.75$. 


\section{DES approach for the pitch-plunge case}

Computations without the transition model show that the flow attains periodicity after the fourth cycle of airfoil motion. However, RANS computations may overpredict the eddy viscosity in the near wall region, which eventually dampens all of the small-scale fluctuations and produces false impression of periodicity in the flowfield and force time history. But DES functions as a sub-grid model in regions where the grid is fine enough, and as a RANS model in regions where it is not.

As seen in Figure 11, PIV and DES results for a random selection (not consecutive) of individual snapshots of the velocity field at $\varphi=0.5$ shows considerable variations; each snapshot differs from the rest, and all differ from the phase average. The implied lack of periodicity in velocity is also clear in the force history, in Figure 12. The time averaged lift coefficient and drag coefficient are 0.88 and 0.07 , respectively, for DES computation. The lift coefficient is comparable to RANS computations with transition but the drag coefficient is larger than in the RANS computations. In all cases the drag coefficient is not far from zero, suggesting that the wake structure would be expected to be near an intermediate case between drag-producing vortex street and thrust producing inverse vortex street [6]. The comparison of time-averaged force coefficients among the three models is summarized in Table 1.

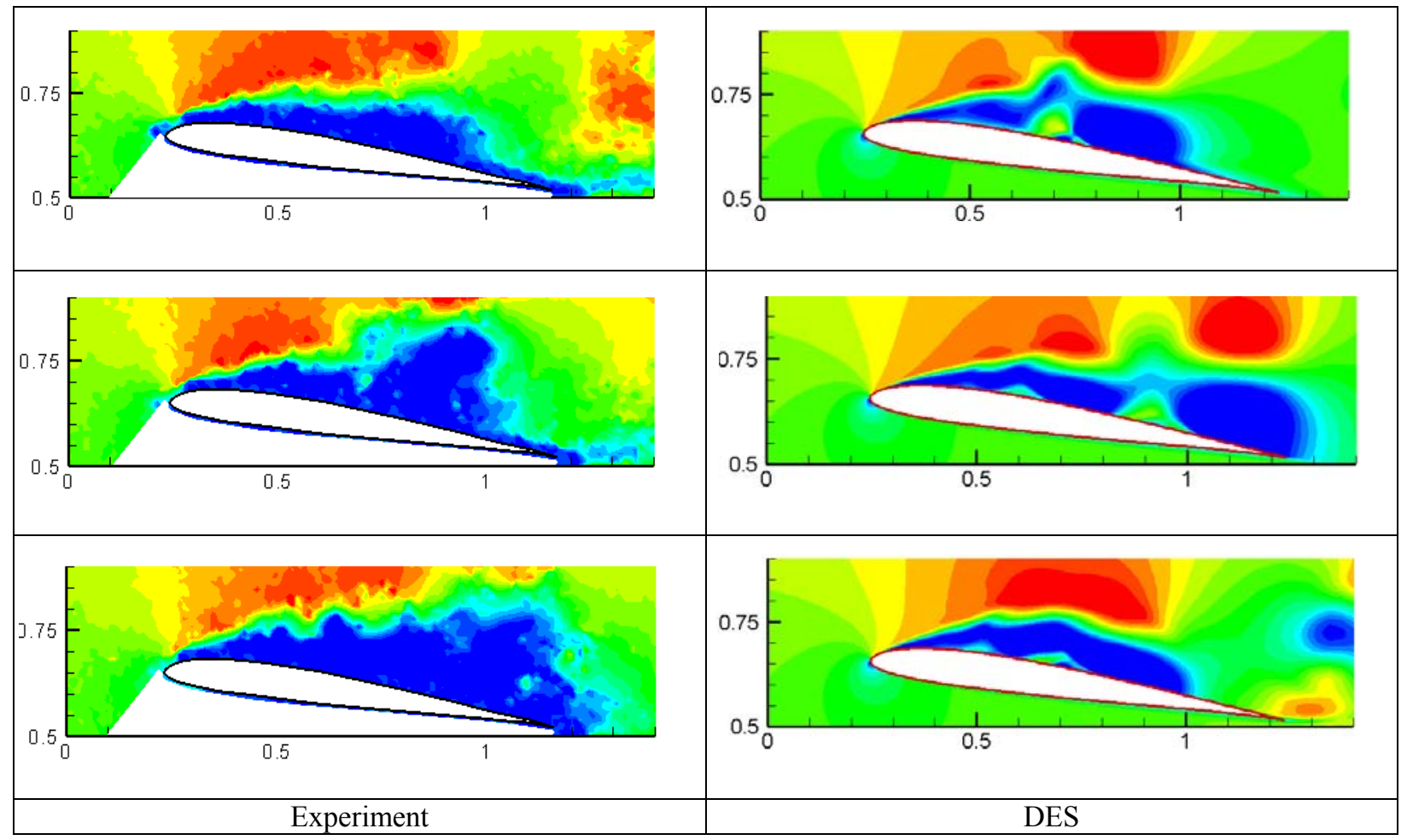

Figure 11. Random selection of individual snapshots of streamwise velocity contours (same contour levels as in Figure 8) at $\varphi=0.5$ for the pitch-plunge case: PIV (left) and DES (right).

Table 1. Time-averaged aerodynamic coefficients with different models

\begin{tabular}{|c|c|c|c|}
\hline & SST without transition & SST with transition model & DES \\
\hline Lift coefficient & 0.80 & 0.85 & 0.88 \\
\hline Drag coefficient & 0.016 & 0.04 & 0.07 \\
\hline
\end{tabular}

DES phase-averaged results (Figure 13) show a separation bubble at $\varphi=0.5$, consistent with the phase-averaged experimental result. But at phase $\varphi=0.25$ DES predicts a large bubble while the experiment does not. The computational phase-average is based on seven cycles of motion, while the experiment uses 230. 

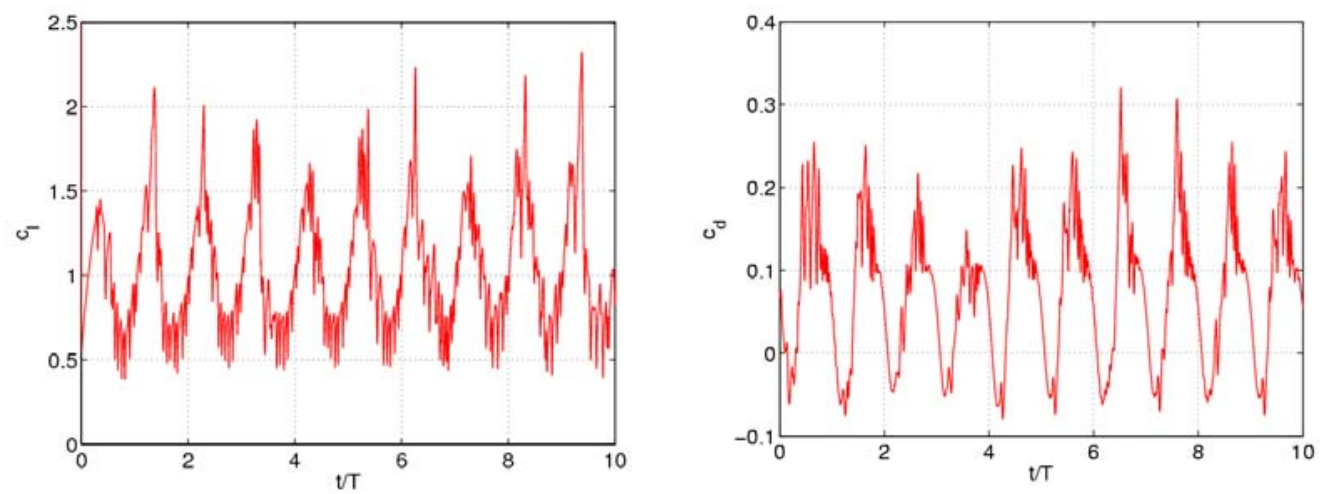

Figure 12. Lift and drag history with DES computations for the pitch-plunge case.
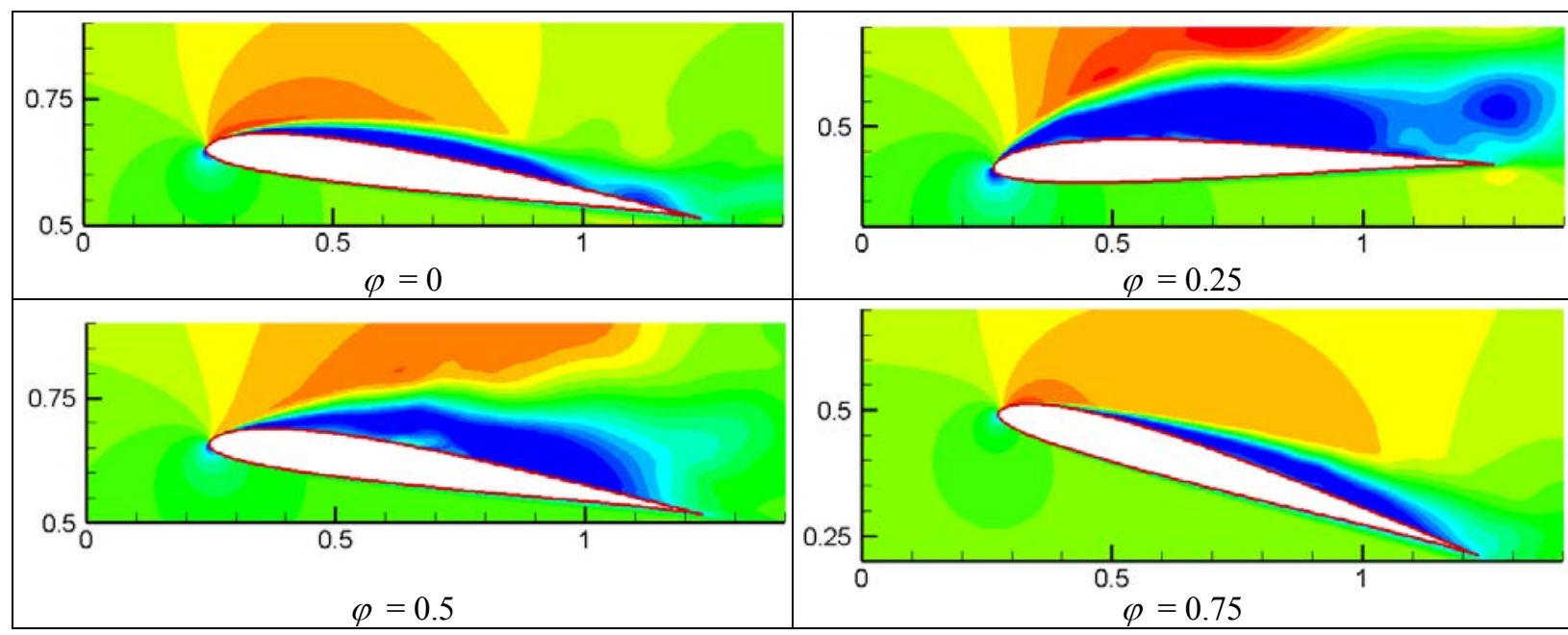

Figure 13. Phase averaged normalized streamwise-component velocity contours with DES for the pitchplunge case.

In the context of SST model, the DES length scale is defined as $\tilde{l}=\min \left(l_{k-\omega}, C_{D E S} \Delta\right)$, and $l_{k-\omega}=k^{1 / 2} /\left(\beta^{*} \omega\right)$. The adjustable parameter $\mathrm{C}_{\mathrm{DES}}$ is chosen with the use of Menter's blending function [22]. In the essence of DES, at the point where the length scale $l_{\mathrm{k}-\omega}$ is much larger than $C_{D E S} \Delta$, the length scale of the model becomes griddependent and the model performs as a subgrid-scale version of the turbulence model. Figure 14 shows at $\varphi=$ 0.5 which regions are modeled and which are computed directly. DES reduces to the SST model in the near-wall regions and regions slightly beyond separation. In most of the separated regions, it functions as a subgrid-scale model.

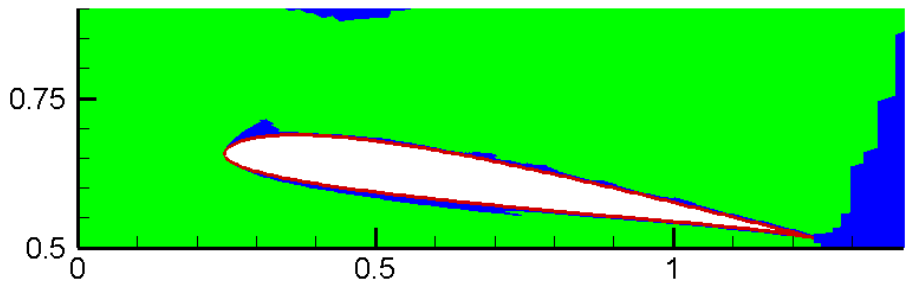

Figure 14. Behavior of DES model: blue regions shown are modeled, and green ones are computed.

Reynolds stress from PIV vs. the computations is compared in Figure 15, again for $\varphi=0.5$. Following prior work on transition in shear layers bounding airfoil separation bubbles in static problems [30], $u^{\prime} v^{\prime}<-0.001$ are of principal interest, with u'v' $=-0.001$ taken as the "threshold" of transition. Overall, the experiment shows much 
larger Reynolds stress values than do the computations. Except for the SST model, no model gives even qualitative agreement with experiment.

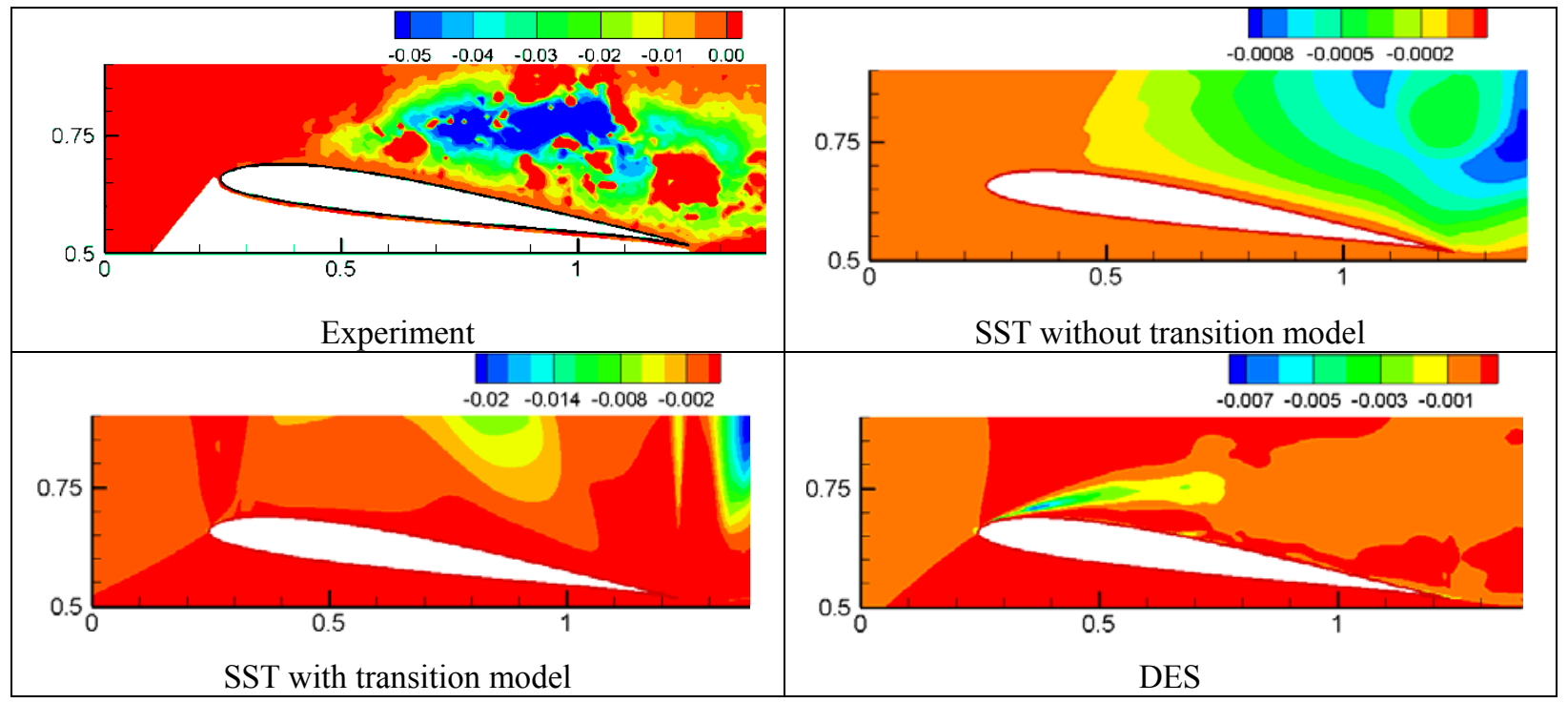

Figure 15. Comparison of the normalized Reynolds stress at $\varphi=0.5$ for pitch-plunge case.

\section{DES approach for the pure-plunge case}

Our second case is pure plunge, where the airfoil maintains a constant geometric angle of attack of $8.42^{\circ}$ and the plunge motion is the same as in the pitch-plunge case discussed above. As shown in Figure 4, the maximum dynamic angle of attack is well beyond the static stall value. DES is used for the computation. The force time histories are shown in Figure 16. The time-averaged lift coefficient is 0.87, and drag coefficient is 0.09; surprisingly, both are comparable to the pitch-plunge case, despite the large difference in angle of attack time history. Though not very clear from the force histories, the velocity contours at the same phase but at different cycles reveal that the flow is not periodic either (Figure 17).
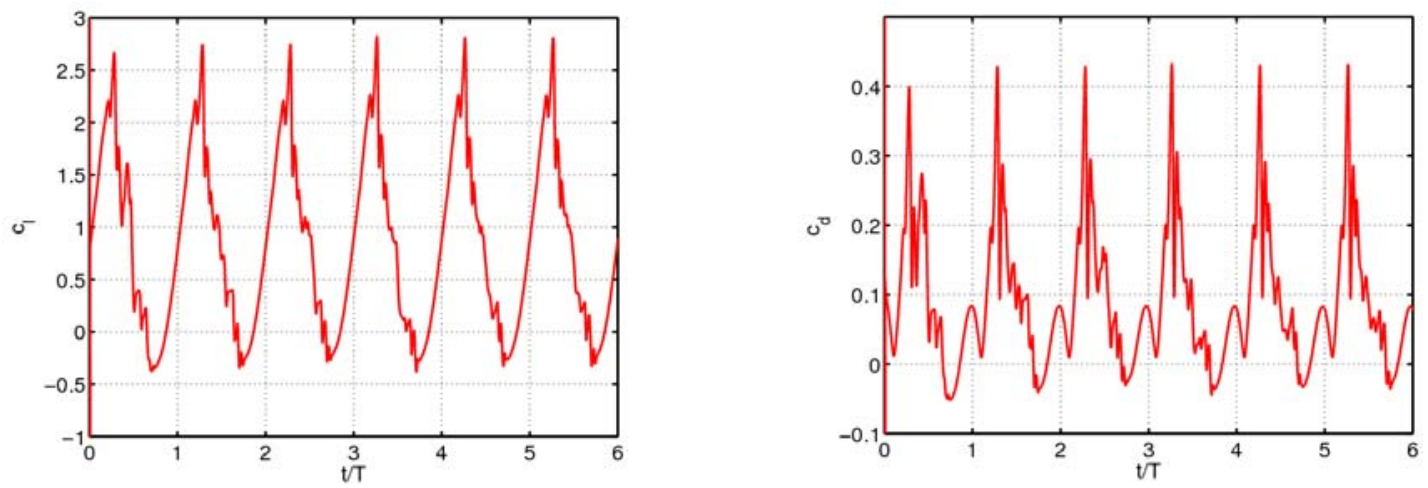

Figure 16. Force histories for the pure-plunge case with DES computations: lift (left) and drag (right).
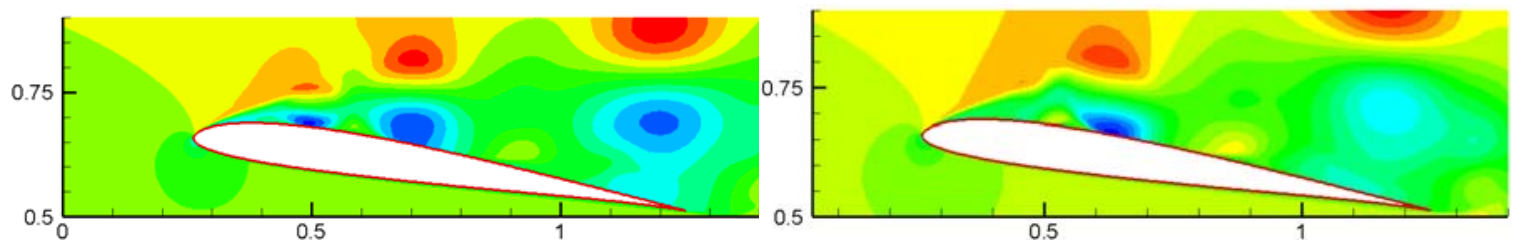

Figure 17. Random snapshots of the normalized velocity contours at $\varphi=0.5$. 
Streamwise-component velocity contours (Figure 18) and out-of-plane component vorticity contours (Figure 19), compare experimental and computational phase-averaged results. For velocity, agreement is good at $\varphi=0$ and 0.25 . At $\varphi=0.5$ the computation gives a stronger shear layer bounding the low-speed separated region over the airfoil suction side, and at $\varphi=0.75$ the computation shows a small separated region near the trailing edge. For vorticity, agreement is best at $\varphi=0$. At $\varphi=0.25$ and 0.5 , computation shows a separated structure akin to a dynamic stall vortex, whereas the experiment gives in the phase average a thin layer of vorticity emanating from the leading edge, with no discernable evidence of strong vortex rollup. Evidently, vortical structures in the experiment are strongly attenuated in the phase average, giving further evidence that the flow is not periodic.

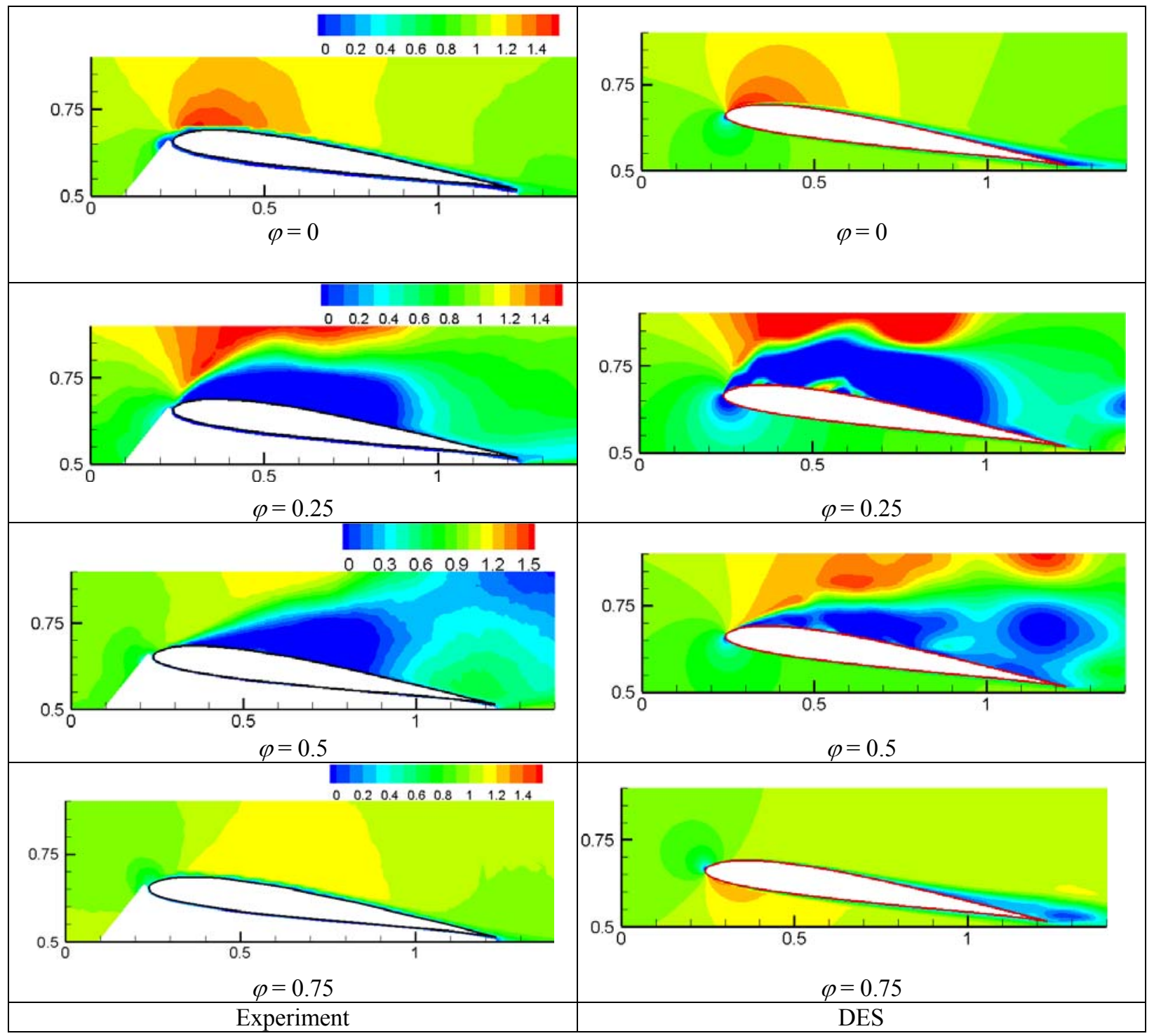

Figure 18. Comparison of normalized streamwise velocity contours at four different phases. 


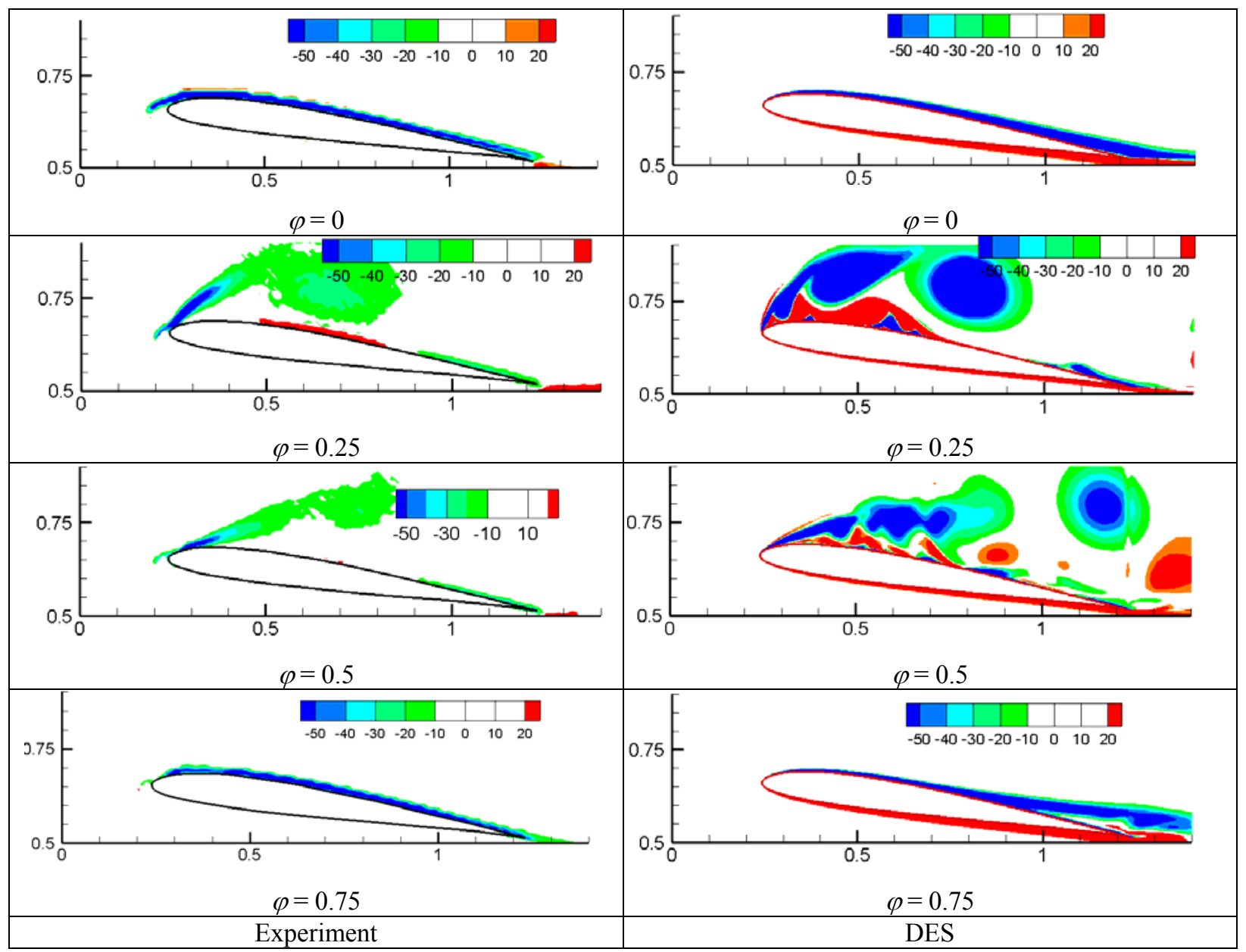

Figure 19. Comparison of normalized out-of-plane vorticity contours at four different phases for the pureplunge case: PIV (left) and DES (right).

\section{Conclusion}

Reasonable qualitative agreement was found in comparison between experiment (particle image velocimetry) and a set of computations (RANS with and without a transition model, and DES) for a SD7003 airfoil in pitch-plunge and in pure-plunge at $\operatorname{Re}=60,000$. For cases where the flowfield is periodic - that is, the phase average over many cycles is similar to individual velocity snapshots - agreement between computation and experiment is especially good. However, in both pitch-plunge and pure-plunge cases the flow markedly departs from periodicity at the bottom of the cosine wave of plunge motion - that is, where the dynamic contribution to total angle of attack is zero, and one quarter phase after the point of maximum dynamic angle of attack. Computations both with and without transition models tend to under-predict Reynolds stress u'v' relative to the experiment. For the pitch-plunge case, streamwise momentum-wake profiles for the RANS computation with transition modeling compare moderately well with the experiment results; other variants of the computations compare worse. Momentum-wake profiles can be loosely taken to imply that the pitch-plunge case is net dragproducing. Computations for time-averaged force show a slight positive drag, but care must be used in relating this to phase-averaged velocity profiles at discrete phases of motion.

\section{Acknowledgement}

This work is partially supported by a Multidisciplinary University Research Initiative (MURI) project sponsored by AFOSR. 


\section{References}

1. Ellington, C. P., "The Aerodynamics of Hovering Insect Flight. I. The Quasi-steady Analysis," Philosophical Transactions of the Royal Society of London, Series B, Vol. 305, Feb. 1984, pp. 1-15.

2. Dickinson, M. H., and Götz, K. G., "Unsteady Aerodynamic Performance of Model Wings at Low Reynolds Numbers," Journal of Experimental Biology, Vol. 174, Jan. 1993, pp. 45-64.

3. Lehmann, F-O., "The Mechanisms of Lift Enhancement in Insect Flight," Naturwissenschaften, Vol. 91, March 2004, pp. 101-122.

4. Liu, H., and Kawachi, K., “A Numerical Study of Insect Flight,” Journal of Computational Physics, Vol. 146, Oct. 1998, pp. 124-156.

5. Shyy, W., Lian, Y., Tang, J., Viieru, D. and Liu, H. Aerodynamics of Low Reynolds Number Flyers, Cambridge University Press, New York, 2008.

6. Koochesfahani, M.M. "Vortical Patterns in the Wake of an Oscillating Airfoil," AIAA J. Vol. 27, No. 9, pp. 1200-1205, Sept. 1989.

7. von Karman, T., and Sears, W. R., "Airfoil Theory for Nonuniform Motion," Journal of the Aeronautical Sciences, Vol. 5, No. 10, 1938, pp. 379-390.

8. McCroskey, W. J., Carr, L. W., and McAlister, K. W., "Dynamic Stall Experiments on Oscillating Airfoils," AIAA J., Vol. 14, No. 1., pp. 57-63, 1976.

9. McCroskey, W. J., “Unsteady Airfoils,” Ann. Rev. Fluid Mech., Vol. 14, 1982, pp. 285-311.

10. Carr, L., "Progress in Analysis and Prediction of Dynamic Stall," J. Aircraft, Vol., 25, 1988, pp. 6-17.

11. Carr, L., and McCroskey, W. J., "A Review of Recent Advances in Computational and Experimental Analysis of Dynamic Stall," IUTAM Symp. On Fluid Dynamics of High Angle of Attack, 1992.

12. Ohmi, K., Coutanceau, M., Loc, T. P., and Dulieu, A., "Vortex Formation around an Oscillating and Translating Airfoil at Large Incidences," J. Fluid Mech., Vol. 211, 1990, pp. 37-60.

13. Ohmi, K., Coutanceau, M., Daube, O., and Loc, T. P., "Further Experiments on Vortex Formation around an Oscillating and Translating Airfoil at Large Incidences," J. Fluid Mech., Vol. 225, 1991, pp. 607-630.

14. Visbal, M., and Shang, J. S., "Investigation of the Flow Structure Around a Rapidly Pitching Airfoil," AIAA J., Vol. 27, No. 8, 1989, pp. 1044-1051.

15. Ghosh Choudhuri, P., and Knight, D. D., "Effects of Compressibility, Pitch Rate, and Reynolds Number on Unsteady Incipient Leading-edge Boundary Layer Separation over a Pitching Airfoil," J. Fluid Mech., Vol. 308, 1996, pp. 195-217.

16. Platzer, M., and Jones, K. "Flapping Wing Aerodynamics - Progress and Challenges" AIAA-2006-500. Jan. 2006

17. Young, J., and Lai, J.C.S. "Oscillation Frequency and Amplitude Effects on the Wake of a Plunging Airfoil". AIAA Journal, Vol. 42, No. 10, 2004. pp. 2042-2052.

18. Lian, Y., and Shyy, W., "Aerodynamics of Low Reynolds Number Plunging Airfoil under Gusty Environment," AIAA Paper 2007-71, Reno, NV, 2007. 
19. http://www.ae.uiuc.edu/m-selig/ads/coord_database.html

20. Anderson, J.M., Streitlien, K., Barrett, D.S. \& Triantafyllou, M.S. (1998) Oscillating foils of high propulsive efficiency, J. Fluid Mech., 360, pp. 41-72.

21. Shyy, W. Computational Modeling for Fluid Flow and Interfacial Transport, 1997, New York, Elsevier.

22. Menter, F. R., "Zonal Two Equation k- $\omega$ Turbulence Models for Aerodynamic Flows,” AIAA Paper 93-2906.

23. Lian, Y., and Shyy, W., "Laminar-Turbulent Transition of a Low Reynolds Number Rigid or Flexible Airfoil," AIAA Journal, Vol. 47, No. 7, 2007. Also AIAA Paper 2006-3051.

24. Smith, A. M. O., and Gamberoni, N., "Transition, Pressure gradient, and Stability Theory," Douglas Aircraft Report ES-26388, 1956.

25. Van Ingen, J. L., "A Suggested Semi-Empirical Method for the Calculation of the Boundary Layer Transition Region,” Inst. of Tech., Dept. of Aeronautics and Engrg., Report VTH-74, Delft, Holland, 1956.

26. Drela, M., and Giles, M. B., "Viscous-Inviscid Analysis of Transonic and Low Reynolds Number Airfoils," AIAA Journal, Vol. 23, No. 10, pp. 1347-1355, 1987.

27. Strelets, M., “Detached Eddy Simulation of Massively Separated Flows,” AIAA paper 01-16694.

28. Peng, S-H, and Eliasson, P., "A Comparison of Turbulence Models in Predication of Flow Around the DLRF6 Aircraft Configuration," AIAA Paper

29. Thomas, P. D., and Lombard, C. K., "Geometric Conservation Law and its Application to Flow Computations on Moving Grids,” AIAA Journal, Vol. 17, No. 10, pp. 1030-1037, 1979.

30. Ol, M., McAuliffe, B. R., Hanff, E. S., Scholz, U., Kaehler, Ch., “Comparison of Laminar Separation Bubble Measurements on a Low Reynolds Number Airfoil in Three Facilities", AIAA 2005-5149, 2005.

31. http://www.cookecorp.com/cooke/php/products/technical 1-en $\quad 01030201 \& v i e w=$ detail\&cam=53.html

32. O1, M. "Vortical Structures in High Frequency Pitch and Plunge at Low Reynolds Number". AIAA 20074233, 2007.

33. Willert, C.E., and Gharib, M. "Digital Particle Image Velocimetry”. Experiments in Fluids, Volume 10, Number 4, January, 1991. 